\title{
Glycosphingolipid GM2 Induces Invasiveness in Irradiation-tolerant Lung Cancer Cells
}

\author{
Seiichiro Ishihara ${ }^{1}$, Kei Aoki ${ }^{1}$, Takeomi Mizutani ${ }^{2}$, Maho Amano ${ }^{3}$, Shin-Ichiro Nishimura ${ }^{3}$, and \\ Hisashi Haga ${ }^{{ }^{*}}$ \\ ${ }^{1}$ Faculty of Advanced Life Science, Hokkaido University, N10-W8, Kita-ku, Sapporo, Hokkaido 060-0810, \\ Japan, ${ }^{2}$ Department of Life Science and Technology, Faculty of Engineering, Hokkai-Gakuen University, \\ 1-1, S26-W11, Chuo-ku, Sapporo, Hokkaido 064-0926, Japan, ${ }^{3}$ Faculty of Advanced Life Science, Hokkaido \\ University, N21-W11, Kita-ku, Sapporo, Hokkaido 001-0021, Japan
}

\begin{abstract}
$A B S T R A C T$. Glycans, including glycosphingolipids, are broadly expressed in plasma membranes and play important roles in cell-cell interactions. Recently, it has been revealed that glycans participate in the regulation of malignant phenotypes of cancer cells, e.g. growth and invasion. However, their roles in irradiation-tolerant cancer cells have not yet been elucidated. In this study, we show that specific glycosphingolipids are highly expressed in invasive, irradiation-tolerant lung cancer cells. Particularly, the glycosphingolipid GM2 contributes to the development of an invasive phenotype in these lung cancer cells. Our results suggest that glycosphingolipids, including GM2, are implicated in the regulation of invasiveness in irradiation-tolerant lung cancer cells and may therefore serve as potential therapeutic targets for lung cancers following radiotherapy.
\end{abstract}

Key words: glycosphingolipids, GM2, invasion, lung cancer cells, radiotherapy

\section{Introduction}

Radiotherapy is one of the most widely accepted therapeutic forms of cancer treatment. However, a portion of cancer cells survive irradiation, resulting in the recurrence of tumors with more malignant phenotypes (von Essen, 1991). Previously, we reported that lung cancer cells that survived irradiation acquire an invasive phenotype (Ishihara et al., 2010, 2015, 2013; Li et al., 2013), which then contributes to cancer progression (Hanahan and Weinberg, 2000). However, the molecular mechanisms that induce invasiveness in these cells have not been fully understood.

Glycans, mainly expressed in plasma membranes of cells, play important roles in cell-cell interactions during immunoreaction and developmental processes by transducing biochemical signals via specific receptors such as galectins (Etulain and Schattner, 2014; Johnson et al.,

\footnotetext{
*To whom correspondence should be addressed: Hisashi Haga, Faculty of Advanced Life Science, Hokkaido University, N10-W8, Kita-ku, Sapporo, Hokkaido 060-0810, Japan.

Tel: +81-11-706-4909, Fax: +81-11-706-3810

Abbreviations: ATF5, activating transcription factor 5; B4GALNT1, beta-1,4 N-acetylgalactosaminyltransferase 1; B3GALT4, beta-1,3galactosyltransferase 4; EMT, Epithelial-mesenchymal transition; GM2A, GM2 activator; GSL, glycosphingolipid; HEXA, Hexosaminidase A; HEXB, Hexosaminidase B; IR cells, irradiation-tolerant P-3 cells; P-3 cells, subclonal parental A549 cells; ST3GAL5, ST3 beta-galactoside alpha-2,3-syalyltransferase 5 .
}

2013). Glycosphingolipids (GSLs) are lipid molecules linked to glycans and are expressed on plasma membranes, where they contribute to intercellular communications (D' Angelo et al., 2013). Notable examples include LacCer, GM3, GM2, and GM1, their expressions being regulated by enzymes such as ST3 beta-galactoside alpha-2,3-syalyltransferase 5 (ST3GAL5), beta-1,4 N-acetylgalactosaminyltransferase 1 (B4GALNT1), beta-1,3-galactosyltransferase 4 (B3GALT4), $\beta$-hexosaminidase A (containing hexosaminidase A (HEXA) and hexosaminidase B (HEXB) subunits), and GM2 activator (GM2A). ST3GAL5, B4GALNT1, and B3GALT4 generate GM3 from LacCer, GM2 from GM3, and GM1 from GM2, respectively (Amado et al., 1998; Harlalka et al., 2013; Nagahori et al., 2013). On the other hand, HEXA and GM2A degrade GM2 to GM3 (Kolter et al., 2005). Recently, several roles of glycans in cancer cells have been reported. For example, glycans biophysically regulate cell-substrate adhesion, and as a result, increase the growth and survival of cancer cells (Paszek et al., 2014). Furthermore, GM2 mediates the migration and invasion of several types of cancer cells (Kundu et al., 2016). However, the detailed roles of glycans, including GSLs, in malignant phenotypes of cancer cells have not yet been elucidated.

Epithelial-mesenchymal transition (EMT), a conversion of cells from an epithelial state to a mesenchymal type, is one of the key events for cells to obtain invasive capacity 
(Friedl and Wolf, 2010; Thiery, 2002). In EMT, cancer cells transform to a mesenchymal state, which triggers loss of cell-cell junctions and apical-basal polarity, cytoskeleton reorganization, changes in the metabolism, and acquisition of invasiveness ( $\mathrm{Li}$ and $\mathrm{Li}, 2015$ ). Mesenchymal cells express specific molecules, including fibronectin, vimentin, and N-cadherin (Zielinska et al., 2015). Therefore, whether cancer cells exhibit a non-invasive epithelial or an invasive mesenchymal phenotype can be determined by assessing their expression of these molecules.

In this study, we revealed that invasive, irradiationtolerant lung cancer cells have higher levels of expression of specific GSLs compared with non-invasive, parental lung cancer cells. Specifically, the GSL GM2 contributes to invasiveness in lung cancer cells and induces the expression of N-cadherin, a marker of mesenchymal phenotypes. Thus, GSLs, including GM2, present potential therapeutic targets for treating lung cancers after radiotherapy.

\section{Materials and Methods}

\section{Cell Culture}

The A549 human lung adenocarcinoma cell line was purchased from American Type Culture Collection (Manassas, VA). Subclonal parental A549 cells (P-3 cells) and irradiation-tolerant P-3 cells (IR cells) were established as we previously reported (Ishihara et al., 2010). The cells were cultured with Dulbecco's modified Eagle medium (Sigma-Aldrich, St. Lois, MO) supplemented with $10 \%$ fetal bovine serum (Biowest, Nuaillé, France) and $1 \%$ antibiotic/antimycotic solution (Sigma-Aldrich). The cells were incubated in a humidified incubator with $5 \% \mathrm{CO}_{2}$ at $37^{\circ} \mathrm{C}$. A type I collagen solution $(0.3 \mathrm{mg} / \mathrm{mL}$; Cell matrix I-C; Nitta Gelatin, Osaka, Japan) was used to prepare a collagen-coated glass dish (Corning, Tewksbury, MA). A type I collagen solution (0.9 $\mathrm{mg} / \mathrm{mL}$; Cell matrix I-P; Nitta Gelatin) was used for making a collagen gel.

\section{Glycoblotting-based GSL profiling}

Cell surface GSLs were profiled quantitatively per the glycoblotting-based protocol reported previously (Gizaw et al., 2015, 2016; Nagahori et al., 2013). In brief, the total lipids extracted from cancer cells $(70-80 \%$ confluent cells on a $10-\mathrm{cm}$ dish) were suspended in $2 \mathrm{~mL} \mathrm{CHCl} / \mathrm{MeOH}(1: 1 \mathrm{v} / \mathrm{v})$ and sonicated. Ozone was generated from oxygen using a benchtop generator at a flow setting of $1 \mathrm{~mL} / \mathrm{min}$. The output from the ozone generator was bubbled through the solution for 5 mins. The reaction was quenched by passing $\mathrm{N}_{2}$ gas through the total lipid suspension for $1 \mathrm{~min}$, and the resulting solution was dried by SpeedVac. $\mathrm{MeOH} / 0.5 \mathrm{M}$ sodium methoxide in $\mathrm{MeOH}(4: 1 \mathrm{v} / \mathrm{v})$ was added to the dried sample and allowed to stand for $30 \mathrm{~min}$ at room temperature for alkaline degradation. The $500 \mu \mathrm{L}$ of reaction mixture was neutralized with $6 \mu \mathrm{L}$ concentrated acetic acid $(\mathrm{AcOH})$ and dried. The sample equivalent of $400 \mu \mathrm{g}$ of protein adjusted with de-ionized water was used for glycoblotting.

BlotGlyco ${ }^{\circledast} \mathrm{H}$ beads $(500 \mu \mathrm{L} ; 10 \mathrm{mg} / \mathrm{mL})$ were added to the wells of MultiScreen Solvinert filter plates, and the water was removed by vacuum. The above mixtures containing whole glycans released from GSLs $(20 \mu \mathrm{L})$ worth $400 \mu \mathrm{g}$ protein and GN4 (500 pmol of chitotetraose) as an internal standard were applied to each well together with $180 \mu \mathrm{L}$ of $2 \% \mathrm{AcOH} /$ Acetonitrile (ACN). The plate was incubated at $80^{\circ} \mathrm{C}$ for 45 mins followed by two successive washings using $200 \mu \mathrm{L}$ of $2 \mathrm{M}$ guanidine- $\mathrm{HCl}$ in $16.6 \mathrm{mM}$ Ammonium bicarbonate $(\mathrm{ABC})$, water, and $1 \%$ triethylamine in $\mathrm{MeOH}$. After acetyl capping using $10 \%$ acetic anhydride in $\mathrm{MeOH}$ at room temperature for $30 \mathrm{~min}$, the remaining acetic anhydride was removed by vacuum. Each well was washed two times with $10 \mathrm{mM}$ hydrochloric acid $(\mathrm{HCl}), \mathrm{MeOH}$, and dioxane, consecutively. ethyl esterification was performed on beads by adding $150 \mathrm{mM} 3$-methyl-1-p-tolyl triazene and incubated at $60^{\circ} \mathrm{C}$ for $90 \mathrm{~min}$. A consecutive washing was performed using $200 \mu \mathrm{L}$ of dioxane, water, $\mathrm{MeOH}$ and water. For an effective transimination reaction, $50 \mathrm{mM}$ benzyloxyamine (BOA) was added, accompanied by $2 \% \mathrm{AcOH} / \mathrm{ACN}$, and incubated at $80^{\circ} \mathrm{C}$ for $45 \mathrm{~min}$. Finally, labelled glycans were eluted with $100 \mu \mathrm{L}$ water.

The glycan moieties of GSLs recovered by glycoblotting were subjected to mass spectrometric analysis after being spotted on MTP 384 target plates (Polished steel TF; Bruker Daltonics, Bremen, Germany) and crystallized together with matrices [DHB:DHB-Na ${ }^{+}$in 9:1]. MALDI-TOF/MS data were acquired on an Ultraflex III (Bruker Daltonics) equipped with a reflector and controlled by the FlexControl 3.0 software. Further fragment analysis was performed by MALDI-TOF/TOF analysis (Bruker Daltonics). Annotation of the MS and TOF/TOF data were achieved with the FlexAnalysis 3.0 software (Bruker Daltonics; $\mathrm{S} / \mathrm{N}=6$, quality factor threshold $=30$ ). The experimental masses were used to predict the possible glycan compositions reported on http:// web.expassy.org/glycomod/ and CFG (http://www.functionalglycomics.org). The data from Lipid Bank (http://lipidbank.jp/) and in-house database were used for the identification of glycans of GSLs.

\section{Immunofluorescence staining}

The cells $\left(4 \times 10^{4}\right)$ were cultured on a collagen-coated hand-made glass dish prepared with a commercial cover glass (Matsunami Glass Ind., Ltd., Osaka, Japan). Two days after seeding, the cells were fixed with $4 \%$ paraformaldehyde (Sigma-Aldrich) in phosphate buffered saline (PBS) and permeabilized with $0.5 \%$ Triton X-100 (Wako Pure Chemical Industries, Ltd., Osaka, Japan) or $0.1 \%$ Saponin (Junsei Chemical Co., Ltd., Tokyo, Japan) in PBS. The cells were blocked with PBS containing $0.5 \%$ bovine serum albumin (BSA; Sigma-Aldrich) for $30 \mathrm{~min}$ at room temperature. Then, the cells were incubated with a primary antibody (1:200, anti-ganglioside GM2 rabbit pAb; Calbiochem, Darmstadt, Germany) in PBS containing 0.5\% BSA at room temperature. After one day, the cells were washed with PBS containing $0.5 \%$ BSA and incubated with a secondary antibody (1:400, Alexa Fluor 594 
Donkey or Goat Anti-Rabbit IgG $(\mathrm{H}+\mathrm{L})$ Antibody; Molecular Probes, Carlsbad, CA) and Alexa Fluor 488 Phalloidin for F-actin staining (1:400; Molecular Probes) or MFP 488 Phalloidin for Factin staining (1:400, MoBiTec $\mathrm{GmbH}$, Göttingen, Germany) in PBS containing $0.5 \% \mathrm{BSA}$ for one hour at $37^{\circ} \mathrm{C}$ or room temperature. Then, the cells were washed with PBS containing $0.5 \%$ BSA and anti-bleaching reagent or PBS were added. The fluorescence images were obtained with a confocal laser scanning microscope (C1 confocal imaging system, Nikon Instech Co., Tokyo, Japan).

\section{$q R T-P C R$}

Cells $\left(3-4 \times 10^{5}\right)$ were seeded on a non-coated plastic dish. Two days after seeding, the cells were lysed with a TriPure Isolation Reagent (Roche, Basel, Switzerland) for RNA extraction. Reverse transcription reaction was performed with a ReverTra Ace qPCR RT Master Mix (TOYOBO, Osaka, Japan). qPCR was performed with a KAPA SYBR FAST qPCR Kit (Kapa Biosystems, Inc., Wilmington, MA). Following primers were used for the experiments: GAPDH, 5'-ACCACAGTCCATGCCATCAC-3' (forward), 5'-TCCACCACCCTGTTGCTGTA-3' (reverse); ST3Gal5, 5'-AGGAAACCCTGCCATTCTGG-3' (forward), 5'-ATGACACCGATTGTGGGGAC-3' (reverse); B4GALNT1 (variant 1 (v1) and variant 2 (v2)), 5'-TGATGCCCAGTAGGGGTTG-3'(forward), 5'-GGCTGTGGGAGAGGTTATGG-3' (reverse); B4GALNT1 (variant 3 (v3)), 5'-CCAGACCAACACAGCAGACAC-3' (forward), 5'-CTCTGCCATTTGCCCACTC-3' (reverse); B3GalT4, 5'-GGTTTTGCACAGCGAGGAAG-3' (forward), 5'-AGGCCACTGCTCCTCTGATA-3' (reverse); HEXA, 5'-ACCAGCGCTACGTCCTTTAC-3' (forward), 5'-TATGCCGTTTCCCTGTGAGG-3' (reverse); HEXB, 5'-GAGTGTGATGCTTTCCCCAAC-3' (forward), 5'-CCTCGTAATGCTCCCCAAAC-3' (reverse); GM2A, 5'-CTTGCCTTGGCTGCATATTTC-3' (forward), 5'-TCCAACAAACCCTTCCAACC-3' (reverse).

\section{$R N A i$}

RNA duplexes (a scramble siRNA and an siRNA targeting GM2A) were synthesized using an in vitro transcription T7 kit (Takara, Otsu, Japan). The sense sequence for the siRNA targeting GM2A was 5'-AACCTTAGAAGTAACTAAAAT-3'. The siRNA duplexes were transfected with a Lipofectamine RNAiMAX Reagent (Invitrogen, Carlsbad, CA).

\section{Spheroid invasion assay}

A spheroid of cells $\left(2 \times 10^{3}\right.$ cells per spheroid) was prepared with Gravity PLUS (InSphero AG, Schlieren, Switzerland). After $48 \mathrm{~h}$ of incubation in a humidified incubator with $5 \% \mathrm{CO}_{2}$ at $37^{\circ} \mathrm{C}$, the spheroid was cultured on a collagen gel-covered glass dish prepared with a commercial cover glass (Matsunami Glass Ind., Lid.). After $1 \mathrm{~h}$ of incubation in a humidified incubator with $5 \%$ $\mathrm{CO}_{2}$ at $37^{\circ} \mathrm{C}$, collagen sol solution was added onto the spheroid and incubated for $30 \mathrm{~min}$ in a humidified incubator with $5 \% \mathrm{CO}_{2}$ at $37^{\circ} \mathrm{C}$. Per this method, the spheroid was cultured in a $3 \mathrm{D}$ colla- gen matrix. After $24 \mathrm{~h}$ of incubation in a humidified incubator with $5 \% \mathrm{CO}_{2}$ at $37^{\circ} \mathrm{C}, \mathrm{F}$-actin staining and capturing the images were performed as described above. The inverse of circularity (perimeter ${ }^{2} /(4 \pi \times$ area $\left.)\right)$ was analyzed with ImageJ software (National Institutes of Health, Bethesda, MD).

\section{Western blotting}

Cells $\left(2 \times 10^{5}\right)$ were seeded on a non-coated plastic dish. One to three days after seeding, cell lysates were prepared by an improved method, as previously reported (Ishihara et al., 2016). Briefly, the cells were fixed with $10 \%$ trichloroacetic acid in PBS for $3 \mathrm{~min}$ on ice and washed three times with PBS on ice. Then, the cells were lysed with SDS sample buffer $(0.25 \mathrm{M}$ Tris- $\mathrm{HCl}$, $5 \%$ of dithiothreitol, $2.3 \%$ of sodium dodecyl sulfate, $10 \%$ of glycerol, and $0.01 \%$ of bromophenol blue; $\mathrm{pH}=6.8$ ). Lysates were sonicated and incubated at $95^{\circ} \mathrm{C}$ for $5 \mathrm{~min}$. The lysates were separated by SDS-PAGE on a $12.5 \%$ polyacrylamide gel $(20 \mathrm{~mA}$ per gel). After blotting to PVDF membranes (Millipore, Billerica, MA), blocking was performed with $5 \%$ skimmed milk or $5 \%$ BSA in TBS-Tween for $60 \mathrm{~min}$ at room temperature. The membranes were then incubated with primary antibodies $(1: 100,000, \mathrm{GAPDH}$ Antibody (6C5) (Ambion, Foster City, CA); 1:10,000, antiB3GALT4 (Abcam, Cambridge, UK); 1:5,000, anti-HEXA (ProteinTech, Chicago, USA); 1:500, anti-GM2A (ProteinTech); 1:2,000, Purified Mouse Anti-N-Cadherin (BD Biosciences, San Jose, CA)) in TBS-Tween at $4^{\circ} \mathrm{C}$ overnight. After washing with TBS-Tween, the membranes were incubated with goat anti-mouse IgG $(\mathrm{H}+\mathrm{L})$-HRP conjugated secondary antibodies $(1: 100,000$ for GAPDH, 1:4,000 for N-cadherin, and 1:5,000 for HEXA and GM2A; BioRad, Hercules, CA) and anti-rabbit IgG, HRPlinkedantibody (1:5,000 for B3GALT4; Cell Signaling Technology, Danvers, MA). After washing with TBS-Tween, signals were detected with an Immobilon Western Chemiluminescent HRP substrate (Millipore). For the detection of HEXA, we used Can Get Signal reagent (TOYOBO). The relative band intensity (Ncadherin/GAPDH) was calculated with ImageJ software (National Institutes of Health) or ChemiDoc Touch (BioRad).

\section{In silico Data analysis of patients}

Kaplan-Meier curves of overall survival in lung cancer patients were analyzed by using the Kaplan-Meier plotter database software (Győrffy et al., 2013) at http://kmplot.com/analysis/index. $\mathrm{php} ? \mathrm{p}=$ service \& cancer $=$ lung.

\section{Statistics}

All of the statistical data were analyzed using Student's $t$-test. 


\section{Results}

\section{GSLs are highly expressed in IR cells}

First, we performed glycoblotting to analyze the total amount of GSLs in parental lung cancer cells (P-3 cells) and irradiation-tolerant lung cancer cells (IR cells). We previously demonstrated that IR cells are a good model of irradiation-tolerant lung cancer cells. Briefly, we generated IR cells by collecting P-3 cells that survived after 10 Gy irradiation (Ishihara et al., 2010). IR cells show high invasion potential in a $3 \mathrm{D}$ collagen matrix dependent on integrins, myosin light chain, and activating transcription factor 5 (ATF5; Ishihara et al., 2010, 2013, 2015). The results of glycoblotting revealed that IR cells expressed approximately twice the amount of total GSLs compared with P-3 cells (Fig. 1A). These results suggest that lung cancer cells that survive irradiation show a higher expression of GSLs than the cells before irradiation.

\section{GM2 is highly expressed in IR cells}

Next, we analyzed the expression of specific GSLs in P-3 and IR cells by glycoblotting. MS analysis revealed that P-3 and IR cells expressed eight kinds of specific GSLs (Fig. 1B, C). Specifically, the expression of one GSL (peak no. 5 in Fig. 1B) was high in P-3 and IR cells (approximately $90 \%$ amount of total GSLs in both cell lines) and was higher in IR cells than in P-3 cells (Fig. 1D). Other GSLs detected by glycoblotting (peaks no. 1 to 4 and 6 to 8 in Fig. 1B) had a higher level of expression in IR cells than in P-3 cells, an exception being the GSL of peak no. 4 (Fig. 1E). Furthermore, we confirmed a detailed structure of the GSL of peak no. 5 by using MS/MS analysis. The results indicated that the GSL is GM2, which contains a GlcCer basic structure, $\mathrm{N}$-acetylgalactosamine, sialic acid, and galactose, in that order (Fig. 1F). Then, we performed immunofluorescence staining to observe GM2 expression in the cells. The proportion of the cells expressing GM2 was greater among IR cells than among P-3 cells (Fig. 2A, B; Fig. S1A, B). These results indicate that IR cells express a higher amount of GM2 than P-3 cells.

We have previously shown that ATF-5 overexpression induces irradiation resistance in P-3 cells (Ishihara et al., 2015). To address whether irradiation induced GM2 expression or selected P-3 cells already expressing high levels of the GSL, we compared GM2 expression in P-3 cells before and after ATF5 overexpression (OE-1). Immunofluorescence staining revealed comparable levels of GM2 expression between OE-1 and the parental P-3 cells (Fig. S1A, B), suggesting that GM2 expression was induced after irradiation of P-3 cells.

\section{IR cells show low expression of B3GALT4 and high expression of HEXA and GM2A}

Because GM2 was highly expressed in IR cells, we next investigated the expression of enzymes contributing to the intracellular generation or degradation of GM2. The mRNA expression of B3GALT4, an enzyme that generates GM1 from GM2, was lower in IR cells than in P-3 cells (Fig. 2C). On the other hand, there were no significant differences in the mRNA expressions of ST3GAL5, which generates GM3 from LacCer, and B4GALNT1, which generates GM2 from GM3, between P-3 and IR cells (Fig. 2C). In addition, the mRNA expression of HEXA and GM2A, which contribute to the degradation of GM2, was higher in IR cells than in P-3 cells (Fig. 2D). On the other hand, the mRNA expression of HEXB, which also contributes to GM2 degradation, was similar in P-3 and IR cells (Fig. 2D). Although the mRNA level of B3GALT4, HEXA, and GM2A was different between IR and P-3 cells, the corresponding protein expression was similar (Fig. 2E). Collectively, the expression of enzymes contributing to the regulation of GM2 expression was not different between P-3 and IR cells.

\section{GM2 promotes invasiveness in lung cancer cells}

Next, we investigated the contribution of GM2 to the invasiveness of lung cancer cells. We previously reported that IR cells exhibit higher invasiveness than P-3 cells in a 3D collagen matrix (Ishihara et al., 2010, 2013, 2015; Li et al., 2013). Because glycoblotting and immunofluorescence staining indicated that IR cells express more GM2 than P-3 cells, we hypothesized that GM2 positively regulates invasiveness in lung cancer cells. Thus, we investigated the invasiveness of P-3 cells with high expression of GM2. To prepare P-3 cells with high expression of GM2, we knocked down GM2A, which is essential for GM2 degradation, by transfecting the cells with an siRNA targeting GM2A. The knock down of GM2A, which increases GM2 expression, was confirmed by qRT-PCR and immunofluorescence staining (Fig. 3A; Fig. S1C, D). To assess the invasiveness of the cells, we performed a spheroid invasion assay in a 3D collagen gel ( $\mathrm{Li}$ et al., 2013). In this method, we analyzed the invasiveness of the cells by examining whether a spheroid invades the surrounding collagen matrix. GM2A-knockdown cells showed a more invasive morphology with more protrusions than control cells (Fig. 3B). Furthermore, the inverse of circularity, which reflects the invasive shape of a spheroid, was higher in GM2Aknockdown cells than in control cells (Fig. 3C). These results suggest that GM2 expression induces an invasive phenotype in lung cancer cells. 

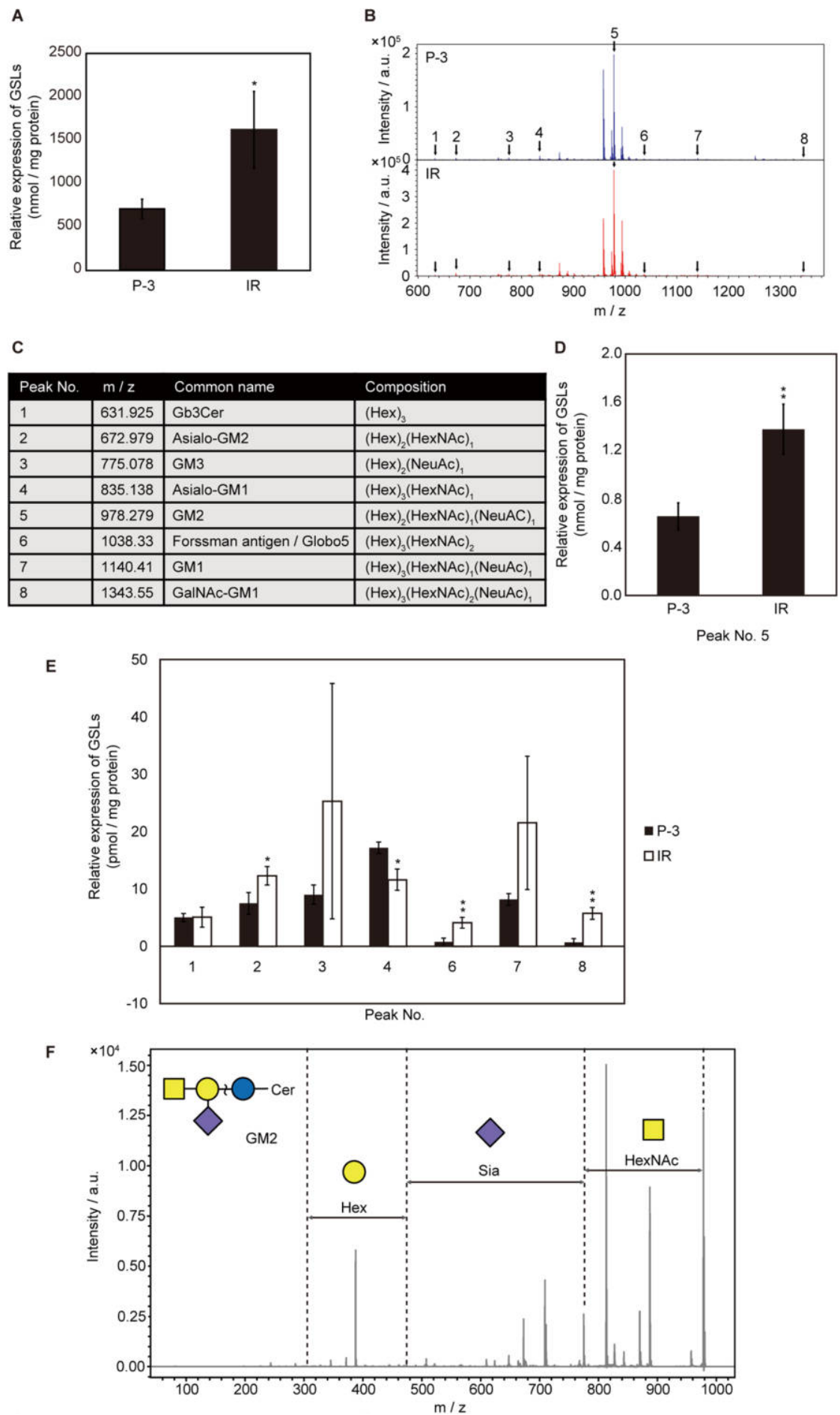

Fig. 1. Expression of glycosphingolipids (GSLs) in lung cancer cells. (A) Total amount of glycosphingolipids in lung cancer cells. The results of glycoblotting in parental lung cancer cells (P-3) and irradiation-tolerant P-3 cells (IR) are shown. (B) The results of MS analysis in P-3 and IR cells. The arrows and numbers show the peaks of specific GSLs. (C) The list of specific GSLs expressed in P-3 and IR cells analyzed from (B). (D) Expression of a GSL (peak no. 5 in (B)) in P-3 and IR cells, as detected by glycoblotting. (E) Expression of GSLs (peaks no. 1 to 4 and 6 to 8 of (B)) in P-3 and IR cells, as detected by glycoblotting. (F) The result of MS/MS analysis in IR cells. Hex, Galactose; HexNAc, N-acetylgalactosamine; Sia, Sialic acid. The cells were cultured on non-coated plastic dishes. $\mathrm{n}=3$ independent experiments. $\mathrm{p}^{*}<0.05, \mathrm{p}^{* *}<0.01$. Error bar $=$ S.D. 

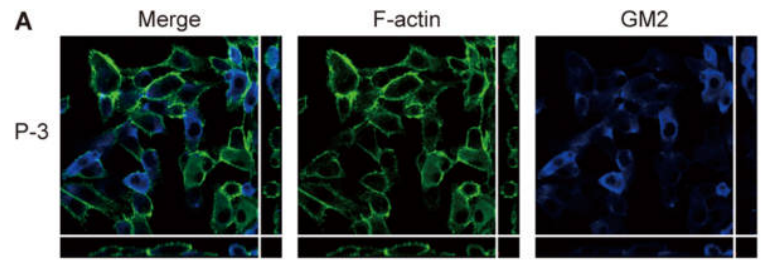

IR
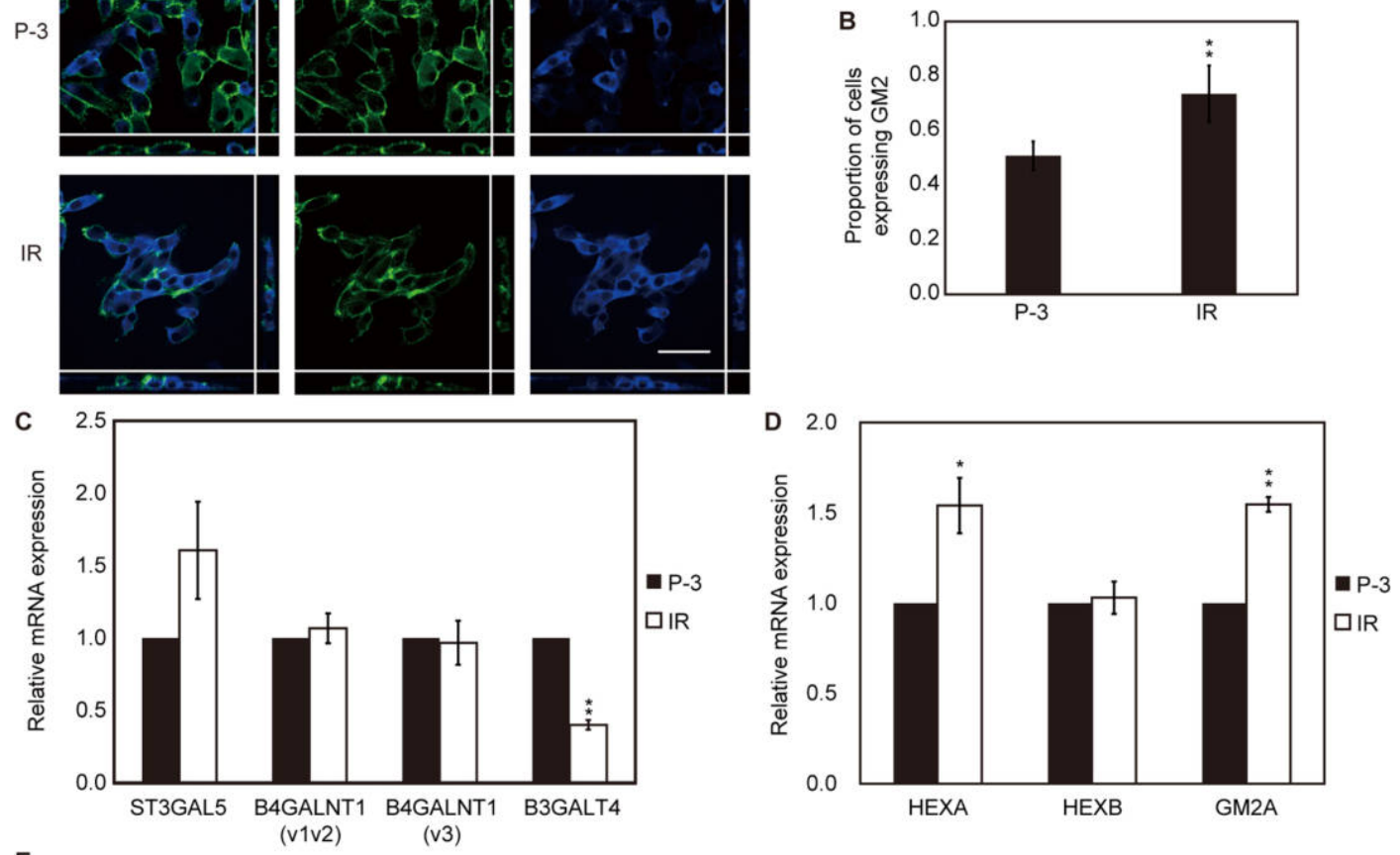

E

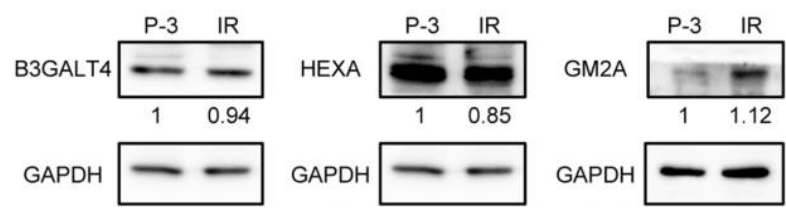

Fig. 2. Expression of GM2 and enzymes generating gangliosides or degrading GM2 in lung cancer cells. (A) The results of immunofluorescence staining targeting GM2 in parental lung cancer cells (P-3) and irradiation-tolerant cells (IR). Cross-sectional views are shown together. Green, F-actin; blue, GM2. The cells were cultured on collagen-coated glass dishes. Bar=50 $\mu \mathrm{m}$. (B) The proportion of cells expressing GM2 calculated from (A). $\mathrm{n}=5$ fields per sample dish. (C) mRNA expression of enzymes that generate gangliosides in parental lung cancer cells (P-3) and irradiation-tolerant cells (IR), as detected by qRT-PCR. The cells were cultured on non-coated plastic dishes. $n=3$ independent experiments. (D) mRNA expression of enzymes that degrade GM2 in P-3 and IR cells, as detected by qRT-PCR. The cells were cultured on non-coated plastic dishes. $\mathrm{n}=3$ independent experiments. (E) Protein expression of enzymes, as detected by western blotting. The number under the blots indicates relative intensity to GAPDH in each protein. $\mathrm{p}^{*}<0.05, \mathrm{p}^{* *}<0.01$. Error bar=S.D.

\section{GM2 enhances $N$-cadherin expression in lung cancer cells}

We analyzed the expression of N-cadherin, a marker of mesenchymal cells. Western blotting revealed that $\mathrm{N}$ cadherin expression is higher in GM2A-knockdown P-3 cells than in control cells, although the difference was not significant (Fig. 3D, E). These results suggest that GM2 promotes EMT in lung cancer cells.

\section{Expression of B3GALT4 and GM2A is correlated with good prognosis}

Finally, we investigated whether the expression of B3GALT4 and GM2A is correlated with prognosis in lung cancer patients. We found that B3GALT4 and GM2A were significantly correlated with good prognosis in lung cancer patients, as the group of patients with high expression of B3GALT4 and GM2A showed better overall survival (Fig. 4A, B). These results are consistent with our hypothesis, as both B3GALT4 and GM2A digest GM2, which increases invasiveness in lung cancer cells.

\section{Discussion}

In this study, we revealed that IR cells, lung cancer cells that survived irradiation, had a higher expression of total GSLs than P-3 cells, their parental cells. Specifically, GM2 expression was greater in IR cells than in P-3 cells. Further- 
A

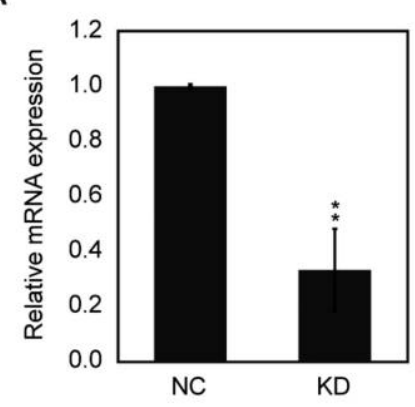

C

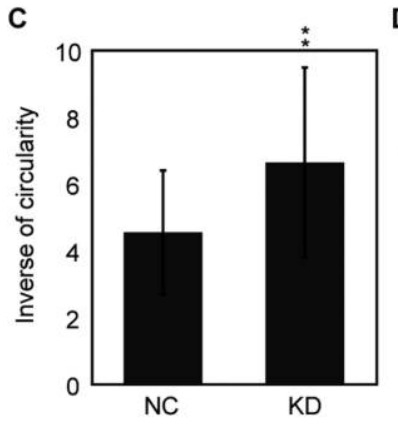

B
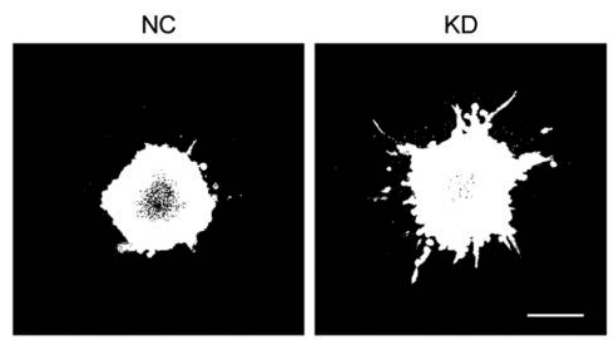

E

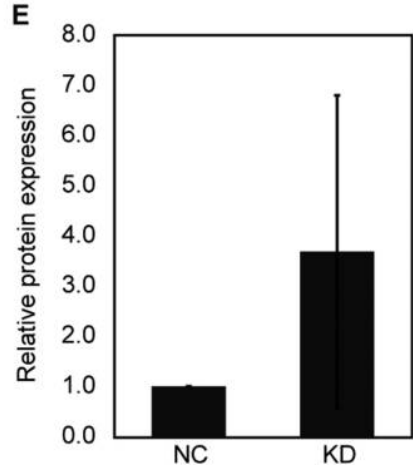

Fig. 3. Contribution of GM2 to invasiveness and N-cadherin expression in lung cancer cells. (A) qRT-PCR analysis of GM2 activator (GM2A), which is essential for GM2 degradation, in parental lung cancer cells transfected with siRNAs. NC, cells transfected with a scramble siRNA as a negative control; $\mathrm{KD}$, cells transfected with siRNA targeting GM2A. $\mathrm{n}=6$ independent experiments. (B) The results of immunofluorescence staining of $\mathrm{F}$-actin in spheroids prepared from the cells. Bar $=200 \mu \mathrm{m}$. (C) The inverse of circularity (perimeter ${ }^{2} /(4 \pi \times$ area)) of spheroids analyzed from (B). The cells were cultured in collagen gel overlay conditions. $\mathrm{n}=40(\mathrm{NC})$ and $49(\mathrm{KD})$ spheroids in 5 independent experiments. (D) Western blotting analysis of N-cadherin and GAPDH as a positive control in parental lung cancer cells transfected with siRNAs. (E) The relative expressions of N-cadherin proteins quantified from (D). The cells were cultured on non-coated plastic dishes. $\mathrm{n}=4$ independent experiments. $\mathrm{p}^{* *}<0.01$. Error bar=S.D.

A

B3GALT4

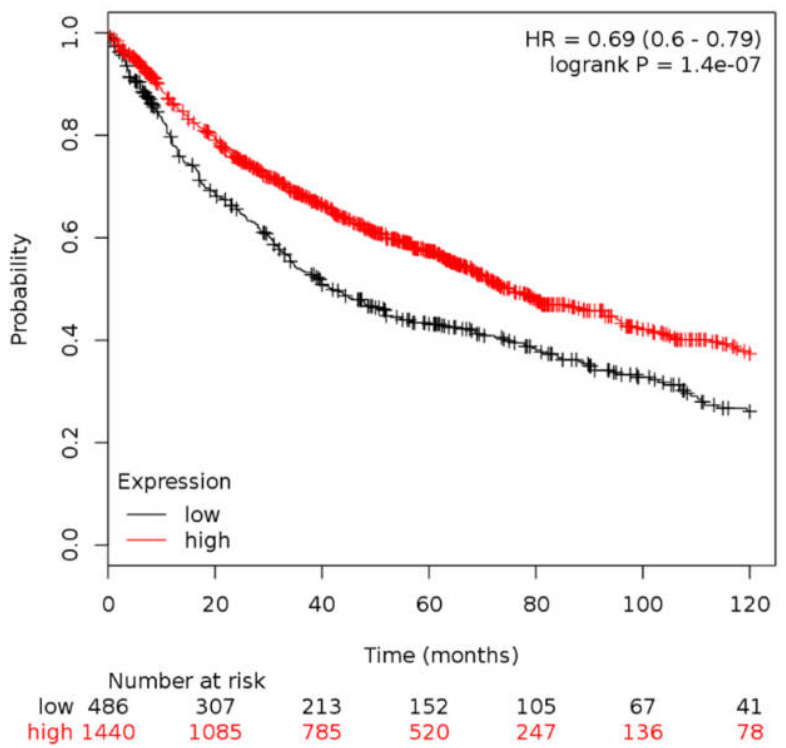

B

\section{GM2A}

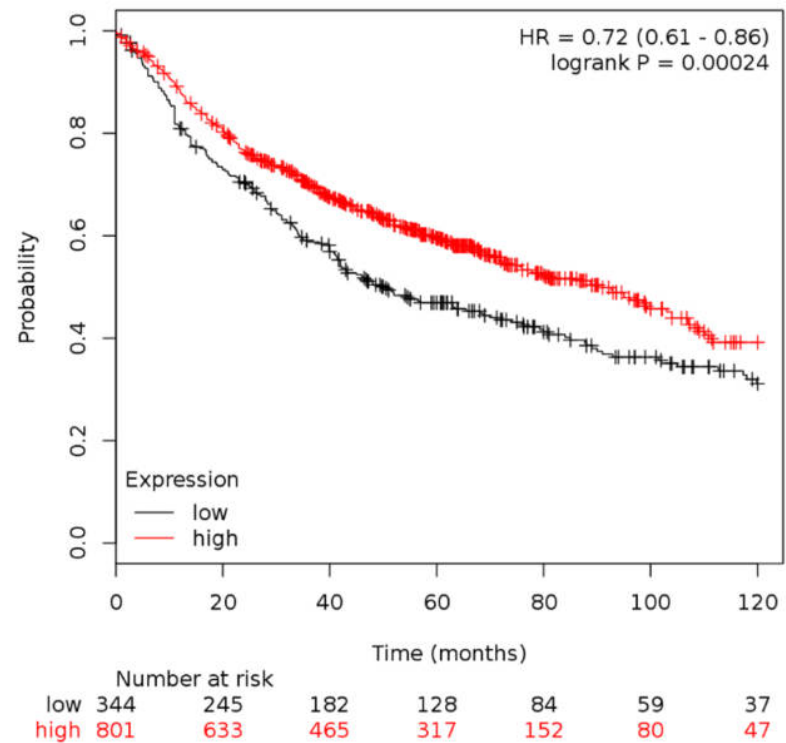

Fig. 4. Kaplan-Meier curves of overall survival in relation to the gene expression of B3GALT4 (A) and GM2A (B). The red and the black curves represent the group with high and low target genes expression, respectively. Hazard ratio (HR) and the values of log-rank P are shown at the top right of the graph. The number of risk indicates the number of patients at the time of the event. 
more, GM2A down-regulation, which enhances GM2 expression, induces high invasiveness and expression of $\mathrm{N}$ cadherin, a marker of mesenchymal cells, in P-3 cells. These results suggest that GM2 induces invasiveness via EMT in lung cancer cells. A recent study showed that GM2 is critical for cancer cell invasion and the expression of vimentin, a key marker of mesenchymal cells (Kundu et al., 2016). We also reported that IR cells show high invasiveness compared with P-3 cells (Ishihara et al., 2010, 2015, 2013; Li et al., 2013). Furthermore, IR cells exhibit a mesenchymal-like morphology in a 3D collagen gel. Thus, IR cells may display high invasiveness via EMT dependent on GM2 expression.

IR cells showed different levels of mRNA expression of enzymes that regulate GM2 expression compared with P-3 cells. We revealed that the mRNA expression of B3GALT4, which generates GM1 from GM2, was lower in IR cells than in P-3 cells, whereas the mRNA expression of ST3GAL5, which generates GM3 from LacCer, and B4GALNT1, which generates GM2 from GM3, was similar in P-3 and IR cells. However, the protein level of B3GALT4 in IR cells was similar to that in P-3 cells. Furthermore, mRNA of HEXA and GM2A, which are essential for GM2 degradation, were highly expressed in IR cells, whereas the mRNA expression of HEXB, which is also critical to GM2 degradation, was similar in P-3 and IR cells. However, we did not observe any significant difference in the protein levels of HEXA and GM2A between IR and P-3 cells. HEXA, HEXB, and GM2A enzymes are all necessary for GM2 degradation (Kolter et al., 2005). We cannot exclude the possibility that other additional enzymes regulate GM2 expression in IR cells.

We suggest that glycans, including the GSL GM2, are potential therapeutic targets for lung cancers following radiotherapy. Previous studies indicate that glycans expressed in plasma membranes regulate the growth and invasiveness of cancer cells (Desiderio et al., 2015; Paszek et $a l ., 2014)$. Moreover, GM2 inhibition by using anti-GM2 antibodies induces cytotoxicity in malignant pleural mesothelioma (Li et al., 2015). These reports suggest that therapeutic approaches targeting glycans are effective for treating malignant tumors. Specifically, in this study, we showed that irradiation-tolerant lung cancer cells highly express GM2. In addition, GM2A-knockdown, which induces GM2 expression, promotes invasiveness in lung cancer cells. Thus, these results suggest that GM2 triggers invasiveness in irradiation-tolerant lung cancer cells. Furthermore, we found that the expression of both B3GALT4 and GM2A, enzymes digest GM2, is correlated with good prognosis in lung cancer. Inhibition of GM2, such as treatment by anti-GM2 antibodies, may be a good therapeutic approach for lung cancer treatment after radiotherapy.
Acknowledgments. This work was supported by JSPS KAKENHI grant numbers $17 \mathrm{~K} 07150$ to H.H.; 25220206 to M.A. and S.N., and JSPS Coreto-Core Program B Asia-Africa Science Platforms to S.N. This research was also supported by AMED under Grant Number JP18gm0810007 to H. $\mathrm{H}$. The authors are grateful to Ms. S. Mori for her technical supports.

\section{References}

Amado, M., Almeida, R., Carneiro, F., Levery, S.B., Holmes, E.H., Nomoto, M., Hollingsworth, M.A., Hassan, H., Schwientek, T., Nielsen, P.A., Bennett, E.P., and Clausen, H. 1998. A family of human beta3galactosyltransferases. Characterization of four members of a UDPgalactose:beta-N-acetyl-glucosamine/beta-nacetyl-galactosamine beta1,3-galactosyltransferase family. J. Biol. Chem., 273: 12770-12778.

D’Angelo, G., Capasso, S., Sticco, L., and Russo, D. 2013. Glycosphingolipids: synthesis and functions. FEBS J., 280: 6338-6353.

Desiderio, V., Papagerakis, P., Tirino, V., Zheng, L., Matossian, M., Prince, M.E., Paino, F., Mele, L., Papaccio, F., Montella, R., Papaccio, G., and Papagerakis, S. 2015. Increased fucosylation has a pivotal role in invasive and metastatic properties of head and neck cancer stem cells. Oncotarget, 6: 71-84.

Etulain, J. and Schattner, M. 2014. Glycobiology of platelet-endothelial cell interactions. Glycobiology, 24: 1252-1259.

Friedl, P. and Wolf, K. 2010. Plasticity of cell migration: a multiscale tuning model. J. Cell Biol., 188: 11-19.

Gizaw, S.T., Koda, T., Amano, M., Kamimura, K., Ohashi, T., Hinou, H., and Nishimura, S. 2015. A comprehensive glycome profiling of Huntington's disease transgenic mice. Biochim. Biophys. Acta, 1850: $1704-1718$.

Gizaw, S.T., Ohashi, T., Tanaka, M., Hinou, H., and Nishimura, S. 2016. Glycoblotting method allows for rapid and efficient glycome profiling of human Alzheimer's disease brain, serum and cerebrospinal fluid towards potential biomarker discovery. Biochim. Biophys. Acta, 1860: $1716-1727$.

Győrffy, B., Surowiak, P., Budczies, J., and Lánczky, A. 2013. Online survival analysis software to assess the prognostic value of biomarkers using transcriptomic data in non-small-cell lung cancer. PLoS One, 8 : e82241.

Hanahan, D. and Weinberg, R.A. 2000. The hallmarks of cancer. Cell, 100: $57-70$.

Harlalka, G.V., Lehman, A., Chioza, B., Baple, E.L., Maroofian, R., Cross, H., Sreekantan-Nair, A., Priestman, D.A., Al-Turki, S., McEntagart, M.E., Proukakis, C., Royle, L., Kozak, R.P., Bastaki, L., Patton, M., Wagner, K., Coblentz, R., Price, J., Mezei, M., Schlade-Bartusiak, K., Platt, F.M., Hurles, M.E., and Crosby, A.H. 2013. Mutations in B4GALNT1 (GM2 synthase) underlie a new disorder of ganglioside biosynthesis. Brain, 136: 3618-3624.

Ishihara, S., Haga, H., Yasuda, M., Mizutani, T., Kawabata, K., Shirato, H., and Nishioka, T. 2010. Integrin beta1-dependent invasive migration of irradiation-tolerant human lung adenocarcinoma cells in 3D collagen matrix. Biochem. Biophys. Res. Commun., 396: 651-655.

Ishihara, S., Yasuda, M., Nishioka, T., Mizutani, T., Kawabata, K., Shirato, H., and Haga, H. 2013. Irradiation-tolerant lung cancer cells acquire invasive ability dependent on dephosphorylation of the myosin regulatory light chain. FEBS Lett., 587: 732-736.

Ishihara, S., Yasuda, M., Ishizu, A., Ishikawa, M., Shirato, H., and Haga, H. 2015. Activating transcription factor 5 enhances radioresistance and malignancy in cancer cells. Oncotarget, 6: 4602-4614.

Ishihara, S., Mizutani, T., Kawabata, K., and Haga, H. 2016. An improved method for western blotting when extracting proteins from mammalian cells cultured on a collagen gel under serum-free conditions. Cytotechnology, 68: 25-32.

Johnson, J.L., Jones, M.B., Ryan, S.O., and Cobb, B.A. 2013. The regula- 
tory power of glycans and their binding partners in immunity. Trends Immunol., 34: 290-298.

Kolter, T., Winau, F., Schaible, U.E., Leippe, M., and Sandhoff, K. 2005. Lipid-binding proteins in membrane digestion, antigen presentation, and antimicrobial defense. J. Biol. Chem., 280: 41125-41128.

Kundu, M., Mahata, B., Banerjee, A., Chakraborty, S., Debnath, S., Ray, S.S., Ghosh, Z., and Biswas, K. 2016. Ganglioside GM2 mediates migration of tumor cells by interacting with integrin and modulating the downstream signaling pathway. Biochim. Biophys. Acta, 1863: 14721489.

Li, L. and Li, W. 2015. Epithelial-mesenchymal transition in human cancer: Comprehensive reprogramming of metabolism, epigenetics, and differentiation. Pharmacology \& Therapeutics, 150: 33-26.

Li, Q., Wang, W., Machino, Y., Yamada, T., Kita, K., Oshima, M., Sekido, Y., Tsuchiya, M., Suzuki, Y., Nan-Ya, K., Iida, S., Nakamura, K., Iwakiri, S., Itoi, K., and Yano, S. 2015. Therapeutic activity of glycoengineered anti-GM2 antibodies against malignant pleural mesothelioma. Cancer Sci., 106: 102-107.

Li, X., Ishihara, S., Yasuda, M., Nishioka, T., Mizutani, T., Ishikawa, M., Kawabata, K., Shirato, H., and Haga, H. 2013. Lung cancer cells that survive ionizing radiation show increased integrin $\alpha 2 \beta 1$ - and EGFRdependent invasiveness. PLoS One, 8: e70905.
Nagahori, N., Yamashita, T., Amano, M., and Nishimura, S. 2013. Effect of ganglioside GM3 synthase gene knockout on the glycoprotein Nglycan profile of mouse embryonic fibroblast. Chembiochem, 14: 7382.

Paszek, M.J., DuFort, C.C., Rossier, O., Bainer, R., Mouw, J.K., Godula, K., Hudak, J.E., Lakins, J.N., Wijekoon, A.C., Cassereau, L., Rubashkin, M.G., Magbanua, M.J., Thorn, K.S., Davidson, M.W., Rugo, H.S., Park, J.W., Hammer, D.A., Giannone, G., Bertozzi, C.R., and Weaver, V.M. 2014. The cancer glycocalyx mechanically primes integrin-mediated growth and survival. Nature, 511: 319-325.

Thiery, J.P. 2002. Epithelial-mesenchymal transitions in tumour progression. Nat. Rev. Cancer, 2: 442-454.

von Essen, C.F. 1991. Radiation enhancement of metastasis: a review. Clin. Exp. Metastasis, 9: 77-104.

Zielinska, H.A., Bahl, A., Holly, J.M., and Perks, C.M. 2015. Epithelialto-mesenchymal transition in breast cancer: a role for insulin-like growth factor I and insulin-like growth factor-binding protein 3? Breast Cancer (Dove Med. Press), 7: 9-19.

(Received for publication, August 10, 2018, accepted, October 12, 2018 and published online, November 8, 2018) 